\title{
The Optimizing Strategies for Prescription of Sleeping Pills for Insomnia Patients
}

\author{
Seockhoon Chung, MD, PhD, Soyoung Youn, MD \\ Department of Psychiatry, Asan Medical Center, University of Ulsan College of Medicine, Seoul, Korea
}

\begin{abstract}
Many patients claim that they cannot sleep well despite ingestion of sleeping pills. In such cases, the physician should explore the reason why sleep disturbance persisted in patients despite administration of sleeping pills. We should consider patients' age, gender, medical or psychiatric diseases, workplace, or sleep environment that may influence patients' sleep and satisfaction with their hypnotics. However, prior to prescribing a hypnotic agent for patients with sleep disturbance, good sleep hygiene education should be provided first. Cognitive-behavioral therapy for insomnia is an important and effective treatment modality to improve patients' sleep quality and reduce dosage of sleeping pills, but it is not easy for most general practitioners to learn and practice this modality. Therefore, we need to consider how to optimize the prescription of hypnotics for treating insomnia in order to prescribe hypnotics safely and appropriately at the lowest dosage. Physician should consider the mechanism of action of each hypnotic agent and prescribe proper hypnotics according to patients' comorbid illness, insomnia types, or other underlying sleep disorders. Also, we should instruct the patients about when they should take their sleeping pills and we should monitor whether they have followed the instructions or not. Hospitalized patients are in a different sleep environment compared to outpatients, and therefore, we should also prepare modified management strategies for inpatients. In this review, strategies for optimizing the prescription of sleeping pills for insomnia patients will be discussed.

Sleep Med Res 2017;8(1):8-16
\end{abstract}

Key Words Optimization, Hypnotics, Insomnia, Sleep.

Received: May 10, 2017

Accepted: May 16, 2017

Correspondence

Seockhoon Chung, MD, PhD

Department of Psychiatry,

Asan Medical Center, University of Ulsan College of Medicine, 88 Olympic-ro 43-gil,

Songpa-gu, Seoul 05505, Korea

Tel +82-2-3010-3411

Fax +82-2-485-8381

E-mailsschung@amc.seoul.kr

\section{INTRODUCTION}

Insomnia is a symptom that is very frequently observed in the clinical field. In most of the cases, it occurs temporarily and improves spontaneously. However, in some cases, it becomes persistent and chronic due to poor sleep hygiene, conditional hyperarousal status, and dysfunctional belief about sleep. Therefore, non-pharmacological treatment to correct poor sleep habit or dysfunctional belief about sleep should be firstly performed before prescribing sleeping pills. ${ }^{1}$ Non-pharmacological treatment can be executed in the form of cognitive-behavioral therapy (CBT) consisting of sleep hygiene, cognitive therapy, sleep restriction, stimulus control and relaxation technique (Table 1). ${ }^{2}$ CBT for insomnia is a very useful and verified tool for insomnia patients, even for their physical health. ${ }^{3}$ However, the physician cannot easily apply CBT to patients, and execution of non-pharmacological therapy takes considerable amount of time and effort. Therefore, sleeping pills are preferentially prescribed in clinical practice to resolve insomnia. Sleeping pills effectively treat insomnia patients in a comparatively short time period. However, such type of pills may cause abuse/dependence problems as well as the risk of falling down or delirium. The use of sleeping pills causes the development of tolerance in insomnia patients, thereby forcing them into long-term use of sleeping pills (Fig. 1). In addition, many patients claim that they cannot sleep well despite ingestion of sleeping pills. In such cases, the dosage of sleeping pills will be increased or sleeping pills will be replaced with other pills. Just like for all other therapies, it is necessary to individualize and optimize the prescription of sleeping pills accordingly to the status and circumstances of the patients. However, to date, no method that can appropriately optimize the prescription of sleeping pills has been established. In this paper, the method for optimizing the pharmaco- 
Table 1. Summary of cognitive-behavioral therapy for insomnia components

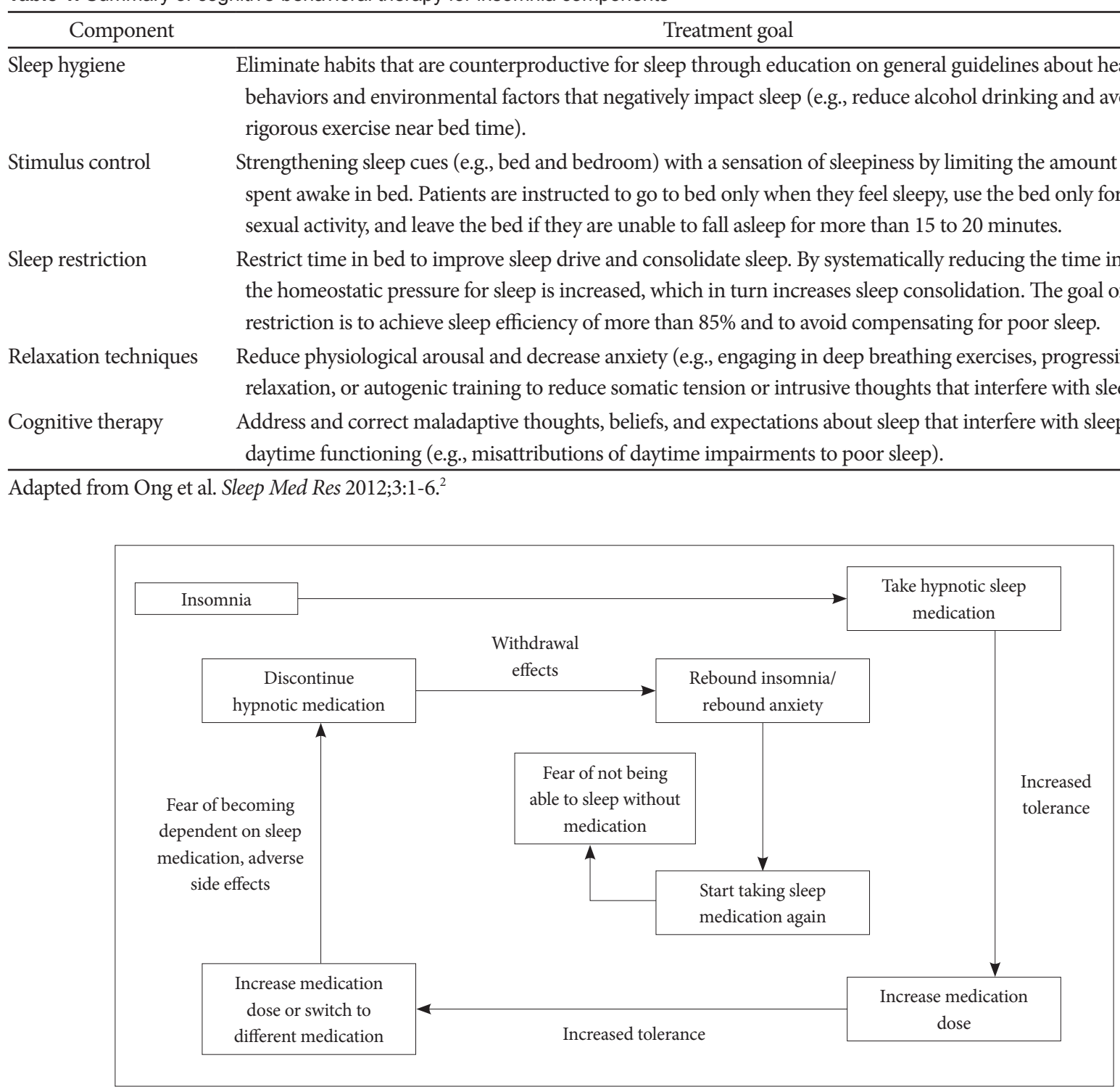

Fig. 1. Difficulty in discontinuing a hypnotic medication in insomnia patients.

logical therapy for insomnia is discussed.

\section{OPTIMIZING PHARMACOLOGICAL TREATMENT FOR INSOMNIA}

Short-term use of sleeping pills is definitely effective in treating insomnia. ${ }^{4}$ However, long-term use of sleeping pills is not recommended, since there is a risk of withdrawal/dependence symptoms. Accordingly, it is necessary for clinicians to establish a method for minimizing and optimizing the prescription of sleeping pills according to the status of their patients.

\section{Exploring Sleep Indices of Insomnia Patients}

When we treat insomnia patients, it is important to explore the sleep structure of each patient. Nocturnal polysomnography is a gold standard tool for assessing the patient's sleep structure, ${ }^{5}$ but it costly and it is not very efficient in assessing the average sleep structure over several days. Actigraphy is another objective assessment tool and it is useful for assessing the sleep structure over several days, ${ }^{6}$ although the averaged-out sleep parameters obtained using actigraphy should be interpreted with caution in some situations. ${ }^{7}$ We can also use a sleep diary, a simple and concise subjective assessment tool. History taking is also useful to understand the patient's sleep structure despite the subjectiveness.

When we assess patients' sleep structure, we can estimate sleep onset latency (SOL), time in bed (TIB), total sleep time (TST), or sleep efficiency (SE) of patients (Table 2). These are the widely used classical sleep indices. Some patients complain of 
Table 2. Sleep indices to explore an individual's sleep structure

\begin{tabular}{lll}
\hline \multicolumn{1}{c}{ Abbreviation } & \multicolumn{1}{c}{ Sleep index } & \multicolumn{1}{c}{ Definition } \\
\hline SOL & Sleep onset latency & $20,30,45$ minutes \\
TIB & Time in bed & 7 hours: 11 pm-6 am \\
TIB d $^{41}$ & Time in bed during 24 hours & $7-8$ hours \\
TST & Total sleep time & $7-8$ hours \\
SE & Sleep efficiency & $85,90 \%$ \\
WASO & Wake after sleep onset & $20,30,45$ minutes \\
PTB $^{8}$ & Duration from administration of pills to bedtime & $<30$ min \\
PTS $^{8}$ & Duration from administration of pills to sleep onset time & $<30$ min \\
PTW $^{8}$ & Duration from administration of pills to wake up time & $7-8$ hours \\
\hline
\end{tabular}

difficulty falling asleep, and others complain of frequent awakening during the night. Increased SOL can provide information on the initial insomnia, and decreased SE indicates that the patient has frequent awakening during sleep. In addition, we propose to use new sleep indices related to the sleeping pill ingestion time ${ }^{8}$ such as duration from administration of sleeping pills to bedtime (PTB), duration from administration of sleeping pills to sleep onset time (PTS), or duration from administration of sleeping pills to wake up time (PTW). We have reported that insomnia patients who were satisfied with their sleeping pills tended to take these pills later in the evening. ${ }^{8}$ Collecting information about the pattern of sleeping pill usage is helpful to provide guidance on when to take sleeping pills. For instance, a particular patient reports that he/she takes sleeping pills within 30 minutes before bedtime although he/she falls asleep 2 hours after sleeping pill ingestion. In this case, we can observe the discrepancy between 30 minutes of PTB and 2 hours of PTS. Therefore, we should assess PTB and PTS separately in patients, and we should provide guidance to patients regarding when they should take sleeping pills for getting better sleep.

\section{Considering Various Factors That Have an Influence on Pharmacological Therapy Effects}

\section{Age and gender}

Various factors, such as patient's age/gender, personality, ${ }^{9,10}$ co-existence of medical disease, sleep environment, workplace and work pattern, have an influence on the pharmacological effects or adverse effects of sleeping pills. In general, it is known that the prevalence of insomnia is higher among patients of higher age, ${ }^{11,12}$ and the proportion of people consuming sleeping pills is higher among the elderly population. ${ }^{13}$ On the other hand, it is recommended that elderly patients with insomnia should take a small dose of sleeping pills because of the higher rates of adverse events. In researches on the usage pattern of sleeping pills in Korea, ${ }^{13}$ it was observed that the rate/frequency of taking sleeping pills is higher and the dosage of sleeping pills is lower among the elderly population. In the elderly population, medical diseases occur more frequently, physical activity levels are decreased, and side effects from various drugs occur frequently. On the other hand, since the metabolic rate gradually decreases among patients of higher age, ${ }^{14}$ it is necessary to reduce the dosage of sleeping pills. In particular, attention should be paid since sleeping pills may cause side effects such as delirium, risk of falling down, and sleep-related eating disorder. $^{15,16}$

The prevalence of insomnia is reported to be higher and the use of sleeping pills is more frequent among females in comparison to males. ${ }^{17,18}$ Women tend to experience more economic/social stress, which may be one of the reasons for sleep disturbance. The prevalence of depressive/anxiety disorders, which are closely associated with sleep disturbance, was reported to be higher among women compared to men. ${ }^{19,20}$ Therefore, especially the presence of comorbid depression or anxiety disorder needs to be assessed when a physician assesses women with sleep disturbance. Conversely, among patients with obstructive sleep apnea (OSA), it was reported that insomnia accompanied by OSA may have negative effects on fatigue and quality of life in men than in women. ${ }^{21}$ Also, aging can have an influence on the severity of OSA among men. ${ }^{22}$

\section{Patients with psychiatric disorders}

It is necessary to precisely examine the underlying stress or psychiatric disorders, which may be the reason for insomnia, prior to prescribing sleeping pills for insomnia symptom. The diagnostic criteria for major depressive disorder include insomnia/hypersomnia, and most of the patients visiting the hospital report the insomnia symptom as chief complaints for their depression. In addition, anxiety disorder tends to frequently accompany insomnia. In particular, some patients tend to control their anxiety using a higher dose of sleeping pills, ${ }^{23}$ when their anxiety symptoms are not well-controlled. In insomnia patients with anxiety disorder, it is more effective to use benzodiazepine hypnotics, rather than z-class drugs, to control their anxiety and sleep at the same time. For the patients with major depressive disorder who suffer from sleep disturbance, we can prescribe $\mathrm{z}$-class drugs or benzodiazepine hypnotics to control their sleep. Another option is to use a sedating antidepressant 
such as trazodone, mirtazapine, or doxepin. Especially, it is necessary to establish a strategy to use mirtazapine instead of sleeping pills in insomnia patients with depression, because mirtazapine has both of strong antidepressant and hypnotic effect. In order to prescribe an antidepressant for patients with depression, we should consider the influence of each antidepressant on sleep. For instance, fluoxetine ${ }^{24}$ may disturb sleep, whereas mirtazapine ${ }^{25}$ may induce sleep.

\section{Patients with medical diseases}

If patients have underlying medical diseases, the insomnia type may vary depending on the characteristics of medical diseases. In addition, it may be necessary to diversify the strategy for selecting sleeping pills depending on the characteristics of medical diseases. For instance, restless legs syndrome (RLS), which can cause initial insomnia, may occur in chronic renal failure patients requiring periodic dialysis. ${ }^{26}$ In addition, since these patients frequently take rest or sleep during the daytime while undergoing dialysis, their circadian rhythm is usually impaired. ${ }^{27}$ In such cases, prescription of sleeping pills is not very effective, and it is necessary to establish a strategy for dealing with the RLS or adjusting the sleep-wake cycle.

If patients have sleep disturbance due to respiratory distress, benzodiazepine hypnotics, which may accelerate dyspnea, ${ }^{28}$ should be used with caution. Rather than using benzodiazepines, we can prescribe z-class drugs or sedating antidepressants such as trazodone or doxepin. ${ }^{29}$ Obese patients usually have difficulty falling into deep sleep due to recurrent sleep apnea during the night. ${ }^{30}$ In such cases, attention should be paid since benzodiazepine hypnotics may accelerate sleep apnea. ${ }^{31}$ The patients who experience pain frequently complain of sleep disturbance. ${ }^{32}$ Although it is necessary to select the approach depending on the reason for the pain, sedating antidepressants such as trazodone or doxepin can be considered since such drugs are effective in decreasing the pain. ${ }^{33}$

\section{Patients with neurologic diseases}

If the patients suffer from accompanying central neurologic diseases, the benzodiazepine drugs or z-class sleeping pills should be prescribed carefully since these drugs may cause delirium. ${ }^{34}$ Depending on the case, an lower dose of antipsychotic medication such as quetiapine, in consultation with psychiatrists, may be more effective and safe. ${ }^{35}$ If the patients suffer from rapid eye movement sleep behavior disorder which is frequently encountered in Parkinson disease, which itself may also be associated with insomnia, ${ }^{36}$ or dementia with Lewy bodies, ${ }^{37} \mathrm{z}$-class sleeping pills are actually not helpful for improving their dream enactment. In such cases, a benzodiazepine such as clonazepam is more effective. ${ }^{38}$

\section{Patients with cancers}

The prevalence of insomnia among cancer patients is report- ed to be higher compared to that in the general population. ${ }^{39}$ When patients are diagnosed with cancer, they may feel depressed and anxious. In addition, hospitalization, chemotherapy, radiation or hormonal therapy may also cause sleep disturbance, and their physical symptoms such as pain, hot flashes, nausea, or vomiting may contribute to the development of insomnia. When cancer patients have sleep disturbance, factors that contribute to insomnia should be explored before prescribing sleeping pills. If a woman with breast cancer cannot fall asleep easily because of her vasomotor symptoms resulting from hormonal therapy, clonidine or venlafaxine $e^{40}$ may be helpful to reduce hot flashes. When pain experienced by cancer patients contributes to the development of insomnia, appropriate pain management should be performed prior to prescribing sleeping pills. Many patients have dysfunctional belief about sleep. i.e., they believe that insomnia is directly related to cancer recurrence or immune dysfunction. A modified CBT for cancer patients to treat their insomnia is currently needed and it should be applied in patients with cancer. ${ }^{41}$ Usually cancer patients spend lot of time lying down on bed due to fatigue symptoms. We propose to use a new sleep index of TIB/d, the time in bed during 24 hours, ${ }^{41}$ to assess the time they spend lying down on bed during the daytime. Prior to prescribing sleeping pills, patients should be educated about a good sleep habit and a proper approach to decrease their fatigue needs to be implemented.

\section{Sleep Disorders as Causes of Sleep Disturbance}

Primary sleep disorders, such as circadian rhythm sleepwake disorder, RLS, and OSA, which may be the causes of sleep disturbance, should be assessed through medical history taking, polysomnography, actigraphy, or rating scales. The circadian rhythm sleep-wake disorder is a condition in which the bedtime or the awakening time is too early (advanced) or too late (delayed), although the TST is usually preserved. Accordingly, in most of the cases, sleeping pills do not provide any additional benefit, and it is rather more effective to use light therapy or administer melatonin for adjusting the sleeping/awakening time. ${ }^{42}$ If the patients suffer from the RLS when they go to sleep, sleeping pills may be helpful to induce sleep. However, a dopamine agonist or clonazepam instead of $\mathrm{z}$-class sleeping pills may show better sleep-inducing effects. ${ }^{43}$ The patients with OSA tend to complain of frequent awakening during the night in spite of falling asleep easily. Also, in some patients who suffer from paradoxical insomnia for a long time, insomnia could occasionally result from the underlying OSA. ${ }^{44}$ Accordingly, for those who have no difficulty in initiating sleep, sleeping pills with short half-lives have no practical effect. In addition, sleeping pills may accelerate OSA, since benzodiazepine hypnotics usually have muscle relaxant effects. ${ }^{45}$ Therefore, it is necessary to apply continuous positive airway pressure or perform a surgical procedure. However, either zolpidem, which has less influence on apnea, ${ }^{46}$ or trazodone,${ }^{47}$ which has a more positive 
Table 3. Drugs that can be prescribed for insomnia in South Korea

\begin{tabular}{ll}
\hline \multicolumn{1}{c}{ Medication } & \multicolumn{1}{c}{ Proposed dosage } \\
\hline Benzodiazepines & $15-30 \mathrm{mg}$ \\
Flurazepam* & $0.125-0.25 \mathrm{mg}$ \\
Triazolam* & $1 \mathrm{mg}$ \\
Flunitrazepam* & $0.25 \mathrm{mg}$ \\
Brotizolam* & $0.5 \mathrm{mg}$ \\
Clonazepam & $5-10 \mathrm{mg}$ \\
Non-benzodiazepine GABA modulators (z-class) & $6.25-12.5 \mathrm{mg}$ \\
Zolpidem IR* & \\
Zolpidem CR* & \\
Antidepressants & $25-50 \mathrm{mg}$ \\
Trazodone & $15-30 \mathrm{mg}$ \\
Mirtazapine & $10-20 \mathrm{mg}$ \\
Amitriptyline & $3-6 \mathrm{mg}$ \\
Doxepin* & \\
Antihistamine & $25 \mathrm{mg}$ \\
Doxylamine* & \\
Melatonin & $2.5-5 \mathrm{mg}$ \\
Prolonged-release melatonin* & \\
Antipsychotics & \\
Quetiapine & \\
Olanzapine & \\
\hline
\end{tabular}

Adapted from Chung et al. J Sleep Med 2016;13:1-7. ${ }^{48}$

*Approved by the Korea Food and Drug Administration.

IR: immediate-release, CR: controlled-release, GABA: gammaaminobutyric acid.

influence on apnea, can be prescribed in selected cases.

\section{Considering the Mechanism of Action of Each Medication}

The ideal hypnotic agent is the one whose sleep-inducing effect begins when the patients go to sleep and the maintenance effect is sustained until the time the patients wake up in the morning. However, the currently available sleeping pills do not have such ideal effects. Therefore, we should select certain hypnotic agents considering their mechanisms of action (Table 3). ${ }^{48}$

\section{Benzodiazepine}

The benzodiazepine has been classically used to improve insomnia through its relaxation/sleep-inducing/anti-anxiety/anti-convulsive effects. Recently, the frequency of prescribing benzodiazepines as hypnotic agents has decreased. However, triazolam, clonazepam, temazepam, flurazepam, and lorazepam are still being frequently used as sleeping pills. Especially, triazolam has been prescribed as a sleep-inducing drug for a long time, with a limitation of 3 weeks in South Korea. The benzodiazepine mainly affects the gamma-aminobutyric acid (GABA) receptor, the GABA-A receptor in particular. Since it affects not only the $\alpha 1$ subunit, but also the $\alpha 2$, $\alpha 3$, and $\alpha 5$ subunits, ${ }^{49}$ it induces sleep-inducing effects along with an additional anxiolytic effect, muscle relaxation, and memory impairment. Attention should be paid since dependence/abuse-related problems may be caused, and muscle relaxation and memory impairment may be directly related to fall and delirium among the elderly population. ${ }^{50}$ On the other hand, benzodiazepine hypnotics are more helpful in some clinical cases where the patients suffer from accompanying anxiety, since the benzodiazepine is more effective in reducing anxiety in comparison to the z-class sleeping pills. ${ }^{51}$

\section{z-class drugs}

In Korea, among the z-class drugs including zolpidem, zopiclone, and zaleplon, it is permitted to prescribe only zolpidem within 28 days. Zolpidem is very effective in inducing sleep since it mainly affects the al subunit of the GABA-A receptor. Therefore, side effects caused by the benzodiazepine family are not caused by zolpidem since it does not affect any other subunits. $^{52}$ Although its sleep-inducing effect has been proved, it relatively lacks the maintenance effect due to its short half-life. The controlled-release (CR) form of zolpidem was developed for this reason. ${ }^{53}$ Since the half-life of the immediate-release (IR) form of zolpidem is short, we can expect lesser daytime functional impairment or somnolence. However, zolpidem must also be cautiously used, since it may cause daytime somnolence, inordinate nighttime behavior, fall, fracture, sleep-related eating disorder, and drowsy driving ${ }^{54}$ against our expectation.

\section{Prolonged-release melatonin}

It has been recently permitted to prescribe prolonged-release melatonin for insomnia patients aged above 55 years in Korea. This drug is manufactured as its secretion is similar to that of intrinsic melatonin. Melatonin is a fat-soluble hormone mainly produced within the pineal gland. It adjusts the individual's circadian rhythm and is involved in the anti-oxidation and immune functions..$^{55}$ The concentration of melatonin is decreased by exposure to light during the day and it gradually increases at night. ${ }^{56}$ Melatonin is regulated by the light entering into the eye and stimulating the suprachiasmatic nucleus through the retinohypothalamic tract. The peak melatonin concentration tends to decrease among the patients of higher age, ${ }^{57}$ and prolongedrelease melatonin was developed to prevent such a decrease in peak concentration, and thereby, induce sleep. It was reported to be free from the dependence/abuse problem, and it can be prescribed for patients who were addicted to the benzodiazepine or z-class hypnotics.

\section{Sedating antidepressants}

Trazodone was initially developed as an antidepressant, but it is currently used as a sleeping pill at a lower dosage. ${ }^{33}$ Its $\mathrm{H}_{1}$ receptor antagonism as well as $5-\mathrm{HT}_{2}$ antagonism is known to 
be related to sleep-inducing and sleep-maintaining effects, and the general dose is considered to be $25-50 \mathrm{mg}$. Doxepin was also used as a tricyclic antidepressant in the past, and now it has been newly developed as a hypnotic agent due to its strong $\mathrm{H}_{1}$ receptor antagonism. It has been reported that doxepin has positive effects on maintaining sleep, ${ }^{58}$ and the general dose is set at 3-6 mg. It has been reported that tricyclic antidepressants may cause side effects such as daytime hypersomnolence, xerostomia, blurred vision, and orthostatic hypotension. However, since the abuse and dependence potential is relatively low, antidepressants can be selected to prevent abuse/dependence-related problems of benzodiazepine hypnotics. ${ }^{59}$ Mirtazapine, a noradrenergic and specific serotonergic antidepressant, can be used when the patients suffer from insomnia accompanied by major depressive disorder. Mirtazapine has a sleep-inducing effect through its $\mathrm{H}_{1}$ receptor antagonism and a sleep-maintaining effect through its $5-\mathrm{HT}_{2 \mathrm{~A} / 2 \mathrm{C}}$ receptor antagonism. ${ }^{60}$ The general dose is set at 7.5-45 mg for antidepressant effects and 30 $\mathrm{mg}$ and below for sleep-inducing effects. However, excessive sedation, increased weight/appetite, and dry mouth were frequently observed. ${ }^{61}$

\section{Antipsychotics}

Antipsychotics are not actually used as sleeping pills, but they are rarely used to induce sleep through their sedative effects. However, they are not recommended for patients with primary insomnia not accompanied by any psychotic symptom, mood symptom, or delirium. Only a smaller doses of antipsychotics, $25-50 \mathrm{mg}$ of quetiapine and $2.5-5 \mathrm{mg}$ of olanzapine, are limitedly used in selected cases.

\section{Selecting Hypnotics Depending on the Types of Sleep Disturbance}

Selection of appropriate sleeping pills plays an important role in optimizing pharmacological treatment of insomnia. Prescribing hypnotics suitable for the insomnia type may prevent not only additional administration of sleeping pills but also unnecessary increase in the dosage of hypnotics.

\section{If the patients cannot fall asleep easily ${ }^{62}$}

The z-class drug, IR form of zolpidem can be primarily selected. If the patient cannot fall asleep easily due to his/her anxiety symptoms, benzodiazepine hypnotics can be selected initially. If the patient cannot fall asleep easily due to delayed circadian rhythm, prolonged-release melatonin can be used to advance their sleep-wake schedule.

\section{If the patients sleep fitfully ${ }^{62}$}

If the patients have no difficulty falling asleep, but they experience frequent awakening during the night, sedating antidepressants such as doxepin and trazodone can be used for maintenance of sleep. In addition, zolpidem CR and long-acting benzodiazepine can be carefully used as well. Prolonged-release melatonin can be used, since it has been reported to enhance the sleep quality.

\section{Evaluating Whether Sleeping Pill Administration Instructions Are Being Accurately Followed}

\section{Benzodiazepine and z-class hypnotics}

It is generally known that sleeping pills should be administered 30 minutes before bedtime. Most of the patients tend to take their prescribed sleeping pills when they want to sleep and not when they should take the medication. To optimize the effects of sleeping pills, hypnotics should be administered when the patients "must sleep" instead of when the patients "want to sleep". According to the results of the research on the association between sleeping pill administration time and sleeping pill satisfaction, ${ }^{8}$ subjects in the satisfied group took their sleeping pills at 23:11 on an average whereas the subjects in the dissatisfied group took their sleeping pills at 21:16 on an average. Between the two groups, there was no significant difference in the time of falling asleep at night and waking up in the morning. In addition, the PTW ${ }^{8}$ was 7.2 hours in the satisfied group and 9.3 hours in the dissatisfied group. This result indicates that earlier administration of sleeping pills from the wake-up time may decrease sleeping pill satisfaction. Based on these results, the authors proposed that the sleeping pill administration guidelines should be revised from "taking sleeping pills 30 minutes before bedtime" to "7 hours prior to waking up in the morning". Actually, the authors also reported that the instruction of taking sleeping pills 7 hours prior to waking up in the morning led to more satisfaction with their pre-existing sleeping pills. ${ }^{63}$ The results of these two researches indicate that the effects of sleeping pills can be maximized when the sleeping pill administration time is optimized for the patients. Also, the physician should monitor whether the patients followed the sleeping pill administration instruction. Only benzodiazepine and z-class sleeping pills were included in these two studies. In the future, it is necessary to conduct for a sedating antidepressant that induces sleep continuity and delta sleep through the antihistaminergic effect and the $5-\mathrm{HT}_{2 \mathrm{~A} / 2 \mathrm{C}}$ receptor-blocking effect.

\section{Prolonged-release melatonin}

The studies conducted in the United Kingdom and France to assess the effects of prolonged-release melatonin reported a slightly low response rate of 26-47\%. ${ }^{64,65}$ Although the response rate might have been underestimated due to the strict definition of the primary outcome, this response rate is still a little low in comparison to the effects expected when sleeping pills are prescribed in general. In order to examine the effects of prolonged-release melatonin itself, we need to assess this effect when the sleep-wake cycle is properly set. In a study assessing the effect of prolonged-release melatonin, the authors reported 
that $66 \%$ of the patients were satisfied with prolonged-release melatonin, and that $44 \%$ of the patients decreased the dosage of their sleeping pills by at least $50 \%$ when it was prescribed for patients who have a regular sleep-wake cycle. ${ }^{66}$ The authors proposed that when prolonged-release melatonin is administered for insomnia patients aged 55 years and above, it is necessary to 1 ) set the sleep-awake cycle to $7-8$ hours, 2 ) make the patients take prolonged-release melatonin 2 hours prior to falling asleep, and 3) make the patients take prolonged-release melatonin for at least 3 weeks in order to enhance the quality of sleep.

\section{Sleep Disturbance in Hospitalized Patients}

Among the hospitalized patients, insomnia is one of the most common complaints. Various medical procedures or laboratory tests can cause the inpatients to suffer from sleep disturbance. Patients' illness, medical disease symptoms, and low physical activity can induce insomnia or circadian rhythm disruption. In South Korea, the multi-bed design of hospitals can also be one of the reasons for sleep disturbance among inpatients. Sixbed rooms, which are very popular in all hospitals in South Korea, can be noisy, crowded, and have impersonal environments compared to single-bed rooms. ${ }^{67,68}$ Hypnotic prescription rate is higher among inpatients ${ }^{69}$ compared to the general population. Numerous sleep-hygiene education or relaxation programs for hospitalized patients have already been performed and the interventions were reported to be effective in reducing sleeping pill prescription rates. ${ }^{70-72}$ However, until now, the inpatient sleep program was focused on educating inpatients about good sleep hygiene. Since doctors order sleeping pills for inpatients in the ward, and occasionally pro re nata (PRN) orders were given by nurses, repeated education of not only the patients but also the medical staff should be performed. We have created and applied a sleep education and sleeping pills reduction program for hospitalized patients (the AMC $i$-sleep program $),{ }^{73}$ including education of not only the patients but also the medical staffs.

Also, physicians usually instruct inpatients to take hypnotics hora somni (HS), which generally means 9:00 pm in most hospitals in South Korea. As shown in the previous study on the association between sleeping pill administration time and patients' subjective satisfaction, ${ }^{8}$ the administration time of sleeping pills among inpatients should be adjusted on an individual basis so as to avoid taking sleeping pills too early.

\section{CONCLUSION}

Although insomnia is a symptom that can be sufficiently improved through adequate diagnosis and treatment, the indiscreet use of sleeping pills causes the patients to develop psychological dependence on sleeping pills. Therefore, the physician should prepare optimizing strategies for pharmacological treatment of insomnia to prevent not only additional administration of sleeping pills but also an unnecessary increase in the dosage of hypnotics. The most important thing is that efforts must be made to examine and improve the cause of insomnia from more diverse perspectives in order to minimize the use of sleeping pills and maximize the therapy satisfaction level through executing the optimized treatment strategies.

\section{Conflicts of Interest}

The authors have no financial conflicts of interest.

\section{REFERENCES}

1. Qaseem A, Kansagara D, Forciea MA, Cooke M, Denberg TD; Clinical Guidelines Committee of the American College of Physicians. Management of chronic insomnia disorder in adults: a clinical practice guideline from the American College of Physicians. Ann Intern Med 2016; 165:125-33.

2. Ong J, Suh S. Utilizing cognitive-behavioral therapy for insomnia to facilitate discontinuation of sleep medication in chronic insomnia patients. Sleep Med Res 2012;3:1-6.

3. Chung S, An H, Park J, Kim H. The effect of non-pharmacological treatment for psychophysiological insomnia on cardiovascular autonomic regulation assessed using heart rate variability. Sleep Med Res 2011;2:10-5

4. Sateia MJ, Buysse DJ, Krystal AD, Neubauer DN, Heald JL. Clinical practice guideline for the pharmacologic treatment of chronic insomnia in adults: an American Academy of Sleep Medicine Clinical Practice Guideline. J Clin Sleep Med 2017;13:307-49.

5. Kushida CA, Littner MR, Morgenthaler T, Alessi CA, Bailey D, Coleman J Jr, et al. Practice parameters for the indications for polysomnography and related procedures: an update for 2005. Sleep 2005;28:499521.

6. Morgenthaler T, Alessi C, Friedman L, Owens J, Kapur V, Boehlecke B, et al. Practice parameters for the use of actigraphy in the assessment of sleep and sleep disorders: an update for 2007. Sleep 2007;30:519-29.

7. Chung S, Youn S, Lee C, Jo MW, Park J, Jo SW, et al. Environmental noise and sleep disturbance: night-to-night variability of sleep/wake pattern. Sleep Med Res 2016;7:78-81.

8. Chung S, Youn S, Yi K, Park B, Lee S. Sleeping pill administration time and patient subjective satisfaction. J Clin Sleep Med 2016;12:57-62.

9. Park JH, An H, Jang ES, Chung S. The influence of personality and dysfunctional sleep-related cognitions on the severity of insomnia. Psychiatry Res 2012;197:275-9.

10. An H, Park J, Jang ES, Chung S. The impact of temperament and character on the efficacy of nonpharmacologic treatment of primary insomnia. Compr Psychiatry 2012;53:201-7.

11. Ohayon MM, Hong SC. Prevalence of insomnia and associated factors in South Korea. J Psychosom Res 2002;53:593-600.

12. Cho YW, Shin WC, Yun CH, Hong SB, Kim J, Earley CJ. Epidemiology of insomnia in Korean adults: prevalence and associated factors. J Clin Neurol 2009;5:20-3.

13. Chung S, Park B, Yi K, Lee J. Pattern of hypnotic drug prescription in South Korea: health insurance review and assessment service-national patients sample. Sleep Med Res 2013;4:51-5.

14. Turnheim K. Drug dosage in the elderly. Is it rational? Drugs Aging 1998;13:357-79.

15. Brodeur MR, Stirling AL. Delirium associated with zolpidem. Ann Pharmacother 2001;35:1562-4.

16. Nzwalo H, Ferreira L, Peralta R, Bentes C. Sleep-related eating disorder secondary to zolpidem. BMJ Case Rep 2013;2013:bcr2012008003.

17. Zhang B, Wing YK. Sex differences in insomnia: a meta-analysis. Sleep 2006;29:85-93. 
18. Stewart R, Besset A, Bebbington P, Brugha T, Lindesay J, Jenkins R, et al. Insomnia comorbidity and impact and hypnotic use by age group in a national survey population aged 16 to 74 years. Sleep 2006;29:1391-7.

19. Piccinelli M, Wilkinson G. Gender differences in depression. Critical review. Br J Psychiatry 2000;177:486-92.

20. Barsky AJ, Peekna HM, Borus JF. Somatic symptom reporting in women and men. J Gen Intern Med 2001;16:266-75.

21. Lee MH, Lee SA, Lee GH, Ryu HS, Chung S, Chung YS, et al. Gender differences in the effect of comorbid insomnia symptom on depression, anxiety, fatigue, and daytime sleepiness in patients with obstructive sleep apnea. Sleep Breath 2014;18:111-7.

22. Chung S, Yoon IY, Lee CH, Kim JW. Effects of age on the clinical features of men with obstructive sleep apnea syndrome. Respiration 2009; 78:23-9.

23. Pourshams M, Malakouti SK. Zolpidem abuse and dependency in an elderly patient with major depressive disorder: a case report. Daru 2014; 22:54.

24. Dorsey CM, Lukas SE, Cunningham SL. Fluoxetine-induced sleep disturbance in depressed patients. Neuropsychopharmacology 1996;14: 437-42.

25. Lawrence RW. Effect of mirtazapine versus fluoxetine on "sleep quality". J Clin Psychiatry 2004;65:1149-50.

26. Stefanidis I, Vainas A, Giannaki CD, Dardiotis E, Spanoulis A, Sounidaki M, et al. Restless legs syndrome and mortality in hemodialysis patients. Sleep Med 2016;22:103.

27. Parker KP, Bliwise DL, Rye DB. Hemodialysis disrupts basic sleep regulatory mechanisms: building hypotheses. Nurs Res 2000;49:327-32.

28. Chen SJ, Yeh CM, Chao TF, Liu CJ, Wang KL, Chen TJ, et al. The use of benzodiazepine receptor agonists and risk of respiratory filure in patients with chronic obstructive pulmonary disease: a nationwide population-based case-control study. Sleep 2015;38:1045-50.

29. Roth T. Hypnotic use for insomnia management in chronic obstructive pulmonary disease. Sleep Med 2009;10:19-25.

30. Luyster FS, Buysse DJ, Strollo PJ Jr. Comorbid insomnia and obstructive sleep apnea: challenges for clinical practice and research. J Clin Sleep Med 2010;6:196-204.

31. Mason M, Cates CJ, Smith I. Effects of opioid, hypnotic and sedating medications on sleep-disordered breathing in adults with obstructive sleep apnoea. Cochrane Database Syst Rev 2015;(7):CD011090.

32. Roberts MB, Drummond PD. Sleep problems are associated with chronic pain over and above mutual associations with depression and catastrophizing. Clin J Pain 2016;32:792-9.

33. Bossini L, Casolaro I, Koukouna D, Cecchini F, Fagiolini A. Off-label uses of trazodone: a review. Expert Opin Pharmacother 2012;13:1707-17.

34. Zaal IJ, Devlin JW, Hazelbag M, Klein Klouwenberg PM, van der Kooi AW, Ong DS, et al. Benzodiazepine-associated delirium in critically ill adults. Intensive Care Med 2015;41:2130-7.

35. Grassi L, Caraceni A, Mitchell AJ, Nanni MG, Berardi MA, Caruso R, et al. Management of delirium in palliative care: a review. Curr Psychiatry Rep 2015;17:550.

36. Chung S, Bohnen NI, Albin RL, Frey KA, Müller ML, Chervin RD. Insomnia and sleepiness in Parkinson disease: associations with symptoms and comorbidities. JClin Sleep Med 2013;9:1131-7.

37. Youn S, Kim T, Yoon IY, Jeong J, Kim HY, Han JW, et al. Progression of cognitive impairments in idiopathic REM sleep behaviour disorder. $J$ Neurol Neurosurg Psychiatry 2016;87:890-6.

38. Devnani P, Fernandes R. Management of REM sleep behavior disorder: an evidence based review. Ann Indian Acad Neurol 2015;18:1-5.

39. Park B, Youn S, Hann CC, Yi K, Lee S, Lee JS, et al. Prevalence of insomnia among patients with the ten most common cancers in South Korea: health insurance review and assessment service-national patient sample. Sleep Med Res 2016;7:48-54.

40. Kligman L, Younus J. Management of hot flashes in women with breast cancer. Curr Oncol 2010;17:81-6.

41. Youn S, Choi B, Yi K, Chung S. Cognitive-behavioral therapy for in- somnia for cacer patients. Korean J Psycho-Oncol. In press 2017.

42. Hardeland R, Poeggeler B, Srinivasan V, Trakht I, Pandi-Perumal SR, Cardinali DP. Melatonergic drugs in clinical practice. Arzneimittelforschung 2008;58:1-10.

43. Shinno H, Oka Y, Otsuki M, Tsuchiya S, Mizuno S, Kawada S, et al. Proposed dose equivalence between clonazepam and pramipexole in patients with restless legs syndrome. Prog Neuropsychopharmacol Biol Psychiatry 2010;34:522-6.

44. An H, Chung S. A case of obstructive sleep apnea syndrome presenting as paradoxical insomnia. Psychiatry Investig 2010;7:75-8.

45. Hanly P, Powles P. Hypnotics should never be used in patients with sleep apnea. J Psychosom Res 1993;37 Suppl 1:59-65.

46. Berry RB, Patel PB. Effect of zolpidem on the efficacy of continuous positive airway pressure as treatment for obstructive sleep apnea. Sleep 2006;29:1052-6.

47. Smales ET, Edwards BA, Deyoung PN, McSharry DG, Wellman A, Velasquez A, et al. Trazodone effects on obstructive sleep apnea and non-REM arousal threshold. Ann Am Thorac Soc 2015;12:758-64.

48. Chung S, Youn S. Optimizing the pharmacological treatment for insomnia. J Sleep Med 2016;13:1-7.

49. Sigel E, Buhr A. The benzodiazepine binding site of GABAA receptors. Trends Pharmacol Sci 1997;18:425-9.

50. French DD, Spehar AM, Campbell RR, Palacios P, Coakley RW, Coblio $\mathrm{N}$, et al. Outpatient benzodiazepine prescribing, adverse events, and costs. In: Henriksen K, Battles JB, Marks ES, Lewin DI. Advances in patient safety: from research to implementation (volume 1: research findings). Rockville, MD: Agency for Healthcare Research and Quality 2005.

51. Hausken AM, Furu K, Skurtveit S, Engeland A, Bramness JG. Starting insomnia treatment: the use of benzodiazepines versus z-hypnotics. A prescription database study of predictors. Eur J Clin Pharmacol 2009; 65:295-301.

52. Rush CR. Behavioral pharmacology of zolpidem relative to benzodiazepines: a review. Pharmacol Biochem Behav 1998;61:253-69.

53. Zammit G. Zolpidem extended-release: therapy for sleep induction and sleep maintenance difficulties. Expert Opin Drug Metab Toxicol 2008;4:325-31.

54. MacFarlane J, Morin CM, Montplaisir J. Hypnotics in insomnia: the experience of zolpidem. Clin Ther 2014;36:1676-701.

55. Altun A, Ugur-Altun B. Melatonin: therapeutic and clinical utilization. Int J Clin Pract 2007;61:835-45.

56. Lewy AJ, Wehr TA, Goodwin FK, Newsome DA, Markey SP. Light suppresses melatonin secretion in humans. Science 1980;210:1267-9.

57. Turek FW, Gillette MU. Melatonin, sleep, and circadian rhythms: rationale for development of specific melatonin agonists. Sleep Med 2004; 5:523-32.

58. Krystal AD, Lankford A, Durrence HH, Ludington E, Jochelson P, Rogowski R, et al. Efficacy and safety of doxepin 3 and $6 \mathrm{mg}$ in a 35-day sleep laboratory trial in adults with chronic primary insomnia. Sleep 2011;34:1433-42.

59. Mendelson WB. A review of the evidence for the efficacy and safety of trazodone in insomnia. J Clin Psychiatry 2005;66:469-76.

60. Rihmer Z, Purebl G. [Mirtazapine--pharmacologic action and clinical advantages]. Neuropsychopharmacol Hung 2009;11:35-40.

61. Matreja PS, Badyal DK, Deswal RS, Sharma A. Efficacy and safety of add on low-dose mirtazapine in depression. Indian J Pharmacol 2012; 44:173-7.

62. Minkel J, Krystal AD. Optimizing the pharmacologic treatment of insomnia: current status and future horizons. Sleep Med Clin 2013;8:333-50.

63. Youn S, Hann CC, Park B, Lee S, Yi K, Chung S. The effects of the new guidance 'take your sleeping pills $7 \mathrm{~h}$ before your wake-up time': a pilot study. Sleep Biol Rhythm 2016;14:397-404.

64. Wade AG, Ford I, Crawford G, McMahon AD, Nir T, Laudon M, et al. Efficacy of prolonged release melatonin in insomnia patients aged 5580 years: quality of sleep and next-day alertness outcomes. Curr Med 
Res Opin 2007;23:2597-605.

65. Lemoine P, Nir T, Laudon M, Zisapel N. Prolonged-release melatonin improves sleep quality and morning alertness in insomnia patients aged 55 years and older and has no withdrawal effects. J Sleep Res 2007;16: $372-80$.

66. Chung S, Youn S, Park B, Lee S, Kim C. The effectiveness of prolongedrelease melatonin in primary insomnia patients with a regular sleepwake cycle. Sleep Med Res 2016;7:16-20.

67. Persson E, Anderberg P, Ekwall AK. A room of one's own--being cared for in a hospital with a single-bed room design. Scand J Caring Sci 2015; 29:340-6.

68. van de Glind I, de Roode S, Goossensen A. Do patients in hospitals benefit from single rooms? A literature review. Health Policy 2007;84:153-61.

69. Youn S, Hann CC, Park B, Lee S, Kim C, Yi K, et al. The sleeping pill prescription rate for inpatients at a general hospital. Sleep Med Res 2016;7:33-8.

70. Youn S, Son JI, Chung S. Preliminary results of sleep pill reduction program for psychiatric inpatients. Korean J Sleep Med 2010;12:19-22.

71. Lareau R, Benson L, Watcharotone K, Manguba G. Examining the feasibility of implementing specific nursing interventions to promote sleep in hospitalized elderly patients. Geriatr Nurs 2008;29:197-206.

72. McDowell JA, Mion LC, Lydon TJ, Inouye SK. A nonpharmacologic sleep protocol for hospitalized older patients. J Am Geriatr Soc 1998; 46:700-5.

73. Youn S, Yi K, Park B, Lee S, Jung YS, Choi JS, et al. Development of sleep-hygiene education and sleeping pills reduction program for hospitalized patients. Korean J Sleep Med 2015;17:33-42. 